\title{
Physics potential of ESS $\nu$ SB in the presence of a light sterile neutrino
}

\author{
Sanjib Kumar Agarwalla, ${ }^{a, b, c}$ Sabya Sachi Chatterjee ${ }^{d, a}$ and Antonio Palazzo ${ }^{e, f}$ \\ ${ }^{a}$ Institute of Physics, Sachivalaya Marg, Sainik School Post, \\ Bhubaneswar 751005, India \\ ${ }^{b}$ Homi Bhabha National Institute, Training School Complex, \\ Anushakti Nagar, Mumbai 400085, India \\ ${ }^{c}$ International Centre for Theoretical Physics, \\ Strada Costiera 11, 34151 Trieste, Italy \\ ${ }^{d}$ Institute for Particle Physics Phenomenology, Department of Physics, Durham University, \\ Durham, DH1 3LE, U.K. \\ e Dipartimento Interateneo di Fisica "Michelangelo Merlin", \\ Via Amendola 173, 70126 Bari, Italy \\ ${ }^{f}$ Istituto Nazionale di Fisica Nucleare (INFN), Sezione di Bari, \\ Via E. Orabona 4, 70126 Bari, Italy \\ E-mail: sanjib@iopb.res.in, sabya.s.chatterjee@durham.ac.uk, \\ palazzo@ba.infn.it
}

ABSTRACT: ESS $\nu$ SB is a proposed neutrino super-beam project at the ESS facility. We study the performance of this setup in the presence of a light eV-scale sterile neutrino, considering $540 \mathrm{~km}$ baseline with 2 years (8 years) of $\nu(\bar{\nu})$ run-plan. This baseline offers the possibility to work around the second oscillation maximum, providing high sensitivity towards $\mathrm{CP}$-violation (CPV). We explore in detail its capability in resolving CPV generated by the standard CP phase $\delta_{13}$, the new CP phase $\delta_{14}$, and the octant of $\theta_{23}$. We find that the sensitivity to $\mathrm{CPV}$ induced by $\delta_{13}$ deteriorates noticeably when going from $3 \nu$ to $4 \nu$ case. The two phases $\delta_{13}$ and $\delta_{14}$ can be reconstructed with a $1 \sigma$ uncertainty of $\sim 15^{0}$ and $\sim 35^{0}$ respectively. Concerning the octant of $\theta_{23}$, we find poor sensitivity in both $3 \nu$ and $4 \nu$ schemes. Our results show that a setup like ESS $\nu \mathrm{SB}$ working around the second oscillation maximum with a baseline of $540 \mathrm{~km}$, performs quite well to explore CPV in $3 \nu$ scheme, but it is not optimal for studying CP properties in $3+1$ scheme.

Keywords: Neutrino Physics, Beyond Standard Model, CP violation

ARXIV EPRINT: 1909.13746 


\section{Contents}

1 Introduction and motivation $\quad 1$

2 Transition probability in the 4-flavor scheme 2

2.1 Theoretical framework 2

2.2 Conversion probability 3

3 Experimental specifications $\quad 4$

4 Details of the numerical analysis $\quad 5$

5 Mass ordering and $\theta_{23}$ octant sensitivity in the 4-flavor scheme $\quad 7$

6 CP-violation searches in the 4-flavor framework $\quad 8$

6.1 Sensitivity to CP-violation 8

$\begin{array}{lll}6.2 & \text { Reconstructing the CP phases } & 12\end{array}$

$\begin{array}{lll}7 & \text { Conclusions and outlook } & 13\end{array}$

\section{Introduction and motivation}

Several anomalous results recorded in short-baseline (SBL) experiments, indicate the existence of a fourth sterile neutrino (for reviews on this subject, see the references [1-6]) with mass $\sim 1 \mathrm{eV}$. The indications come from the accelerator experiments LSND [7] and MiniBooNE [8], and from the so-called reactor [9] and Gallium [10, 11] anomalies. Constraints on light sterile neutrinos have been derived also by the long-baseline (LBL) experiments MINOS and MINOS+ $[12,13], \mathrm{NO} \nu \mathrm{A}[14]$ and T2K [15], by the reactor experiments Daya Bay [16], ${ }^{1}$ DANSS [18] and NEOS [19], by the atmospheric neutrino data collected in Super-Kamiokande [20], IceCube [21] and ANTARES [22], and by solar neutrinos [23-25].

New SBL experiments are under construction, with the aim of testing this intriguing hypothesis (see the review in [26]). The new SBL experiments are sensitive to the characteristic $L / E$ dependency due to the oscillations intervening at the new mass-squared splitting. This will allow them to measure with precision the value $\Delta m_{\text {new }}^{2} \sim 1 \mathrm{eV}^{2}$ and the new mixing angles of the sterile sector. However, as already stressed in the literature, the SBL experiments will be unable to furnish any information about the CP-violation (CPV) structure of the sterile sector. Even the simplest extended framework involving only one neutrino state, the so-called $3+1$ scheme, entails two additional $\mathrm{CP}$ phases with respect

\footnotetext{
${ }^{1}$ We also mention the work [17], where the combination of MINOS, Daya-Bay, and Bugey-3 was considered.
} 
to the standard framework. Therefore, after a hypothetical discovery made at the SBL experiment, we will face the problem of finding a way to determine these new $\mathrm{CP}$ phases.

In order to measure any $\mathrm{CP}$ phase one must be sensitive to the quantum interference of two different oscillation frequencies. In the $3+1$ scenario, in SBL experiments, only the new frequency is observable, while both the standard (solar and atmospheric) frequencies have no effect at all. For this reason the SBL setups have no sensitivity to CPV (both in 3 -flavor and $3+1$ schemes). As first shown in [27], things are qualitatively different in LBL setups, since in these experiments the interference between two different frequencies becomes observable. In fact, the LBL experiments are able to detect both the effects of the standard CP phase and those of the new ones. For this reason, the LBL experiments are complementary to the SBL ones in nailing down the properties of sterile neutrinos.

The new-generation of LBL experiments [28-37] are designed to have a central role in the search of CPV phenomena. In this paper, we focus on the proposed super beam experiment to be performed at the European Spallation Source (ESS $\nu$ SB). This facility will have a very powerful neutrino beam with an average power of $5 \mathrm{MW}$, and the flux is expected to peak around $0.25 \mathrm{GeV}$. We assume that these neutrinos will travel a distance of $540 \mathrm{~km}$ providing the opportunity to work around the second oscillation maximum.

Our present study is complementary to other recent investigations performed about DUNE [38-44], T2HK [44-46], and T2HKK [44, 47]. Other studies on the impact of light sterile neutrinos in LBL setups can be found in [48-56]. We underline that while our study deals with charged current interactions, one can obtain valuable information on active-sterile oscillations parameters also from the analysis of neutral current interactions (see [12-15] for constraints from existing data and $[43,57]$ for sensitivity studies of future experiments.)

The paper has the following structure. In section 2, we detail the theoretical framework and also describe the properties of the 4 -flavor $\nu_{\mu} \rightarrow \nu_{e}$ transition probability. In section 3 , the $\mathrm{ESS} \nu \mathrm{SB}$ setup is described in detail. In section 4 we present the details of our numerical study. In section 5 we briefly explain the (lack of) sensitivity to the neutrino mass hierarchy and to the octant of $\theta_{23}$ making use of the bievents plots. In section 6 we describe the sensitivity to $\mathrm{CPV}$ and the ability to reconstruct the CP phases. Finally, we trace the conclusions in section 7 .

\section{Transition probability in the 4-flavor scheme}

\subsection{Theoretical framework}

In the enlarged $3+1$ framework, the connection among the flavor $\left(\nu_{e}, \nu_{\mu}, \nu_{\tau}, \nu_{s}\right)$ and the mass eigenstates $\left(\nu_{1}, \nu_{2}, \nu_{3}, \nu_{4}\right)$ is provided by a $4 \times 4$ unitary matrix

$$
U=\tilde{R}_{34} R_{24} \tilde{R}_{14} R_{23} \tilde{R}_{13} R_{12},
$$

where $R_{i j}\left(\tilde{R}_{i j}\right)$ represents a real (complex) $4 \times 4$ rotation of a mixing angle $\theta_{i j}$ which contains the $2 \times 2$ submatrix

$$
R_{i j}^{2 \times 2}=\left(\begin{array}{cc}
c_{i j} & s_{i j} \\
-s_{i j} & c_{i j}
\end{array}\right), \quad \tilde{R}_{i j}^{2 \times 2}=\left(\begin{array}{cc}
c_{i j} & \tilde{s}_{i j} \\
-\tilde{s}_{i j}^{*} & c_{i j}
\end{array}\right),
$$


in the $(i, j)$ sub-block. For brevity we have defined

$$
c_{i j} \equiv \cos \theta_{i j}, \quad s_{i j} \equiv \sin \theta_{i j}, \quad \tilde{s}_{i j} \equiv s_{i j} e^{-i \delta_{i j}} .
$$

The parametrization in eq. (2.1) is particularly advantageous because: i) The 3 -flavor expression is recovered by setting $\theta_{14}=\theta_{24}=\theta_{34}=0$. ii) For small values of the mixing angles $\theta_{14}, \theta_{24}$, and $\theta_{13}$, it is $\left|U_{e 3}\right|^{2} \simeq s_{13}^{2},\left|U_{e 4}\right|^{2}=s_{14}^{2},\left|U_{\mu 4}\right|^{2} \simeq s_{24}^{2}$, and $\left|U_{\tau 4}\right|^{2} \simeq s_{34}^{2}$, implying a clear physical meaning of the three mixing angles. iii) Positioning the matrix $\tilde{R}_{34}$ in the leftmost location ensures that the $\nu_{\mu} \rightarrow \nu_{e}$ conversion probability in vacuum is independent of $\theta_{34}$ and of the related CP phase $\delta_{34}$ (see [27]).

\subsection{Conversion probability}

For the $\operatorname{ESS} \nu \mathrm{SB}$ baseline $(540 \mathrm{~km})$, matter effects are very small. This allows us to limit the discussion to the case of propagation in vacuum. As first shown in [27], the $\nu_{\mu} \rightarrow \nu_{e}$ the conversion probability is the sum of three contributions

$$
P_{\mu e}^{4 \nu} \simeq P^{\mathrm{ATM}}+P_{\mathrm{I}}^{\mathrm{INT}}+P_{\mathrm{II}}^{\mathrm{INT}} .
$$

The first term is positive definite and depends on the atmospheric mass-squared splitting. It provides the leading contribution to the transition probability. The expression of this term is given by

$$
P^{\mathrm{ATM}} \simeq 4 s_{23}^{2} s_{13}^{2} \sin ^{2} \Delta,
$$

where $\Delta \equiv \Delta m_{31}^{2} L / 4 E$ is the (atmospheric) oscillating factor, $L$ and $E$ being the neutrino baseline and energy, respectively. The other two terms in eq. (2.4) are induced by the interference of two different frequencies and are not positive definite.. The second term in eq. (2.4) is related to the interference of the solar and atmospheric frequencies and can be expressed as

$$
P_{\mathrm{I}}^{\mathrm{INT}} \simeq 8 s_{13} s_{12} c_{12} s_{23} c_{23}(\alpha \Delta) \sin \Delta \cos \left(\Delta+\delta_{13}\right) .
$$

It should be noticed that at the first (second) oscillation maximum one has $\Delta \sim \pi / 2$ $(\Delta \sim 3 \pi / 2)$. For this reason, in $\mathrm{ESS} \nu \mathrm{SB}$, which works at the second oscillation maximum, one expects an enhanced sensitivity to the CP phase $\delta_{13}$. Indeed, in spite of the lower statistics, we will see how ESS $\nu \mathrm{SB}$ can attain a sensitivity similar to that obtained in the higher statistics experiment T2HK, which works at the first oscillation maximum. The third term in eq. (2.4) appears as a new genuine 4-flavor effect, and is connected to the interference of sterile and atmospheric frequencies. It can be written in the form [27]

$$
P_{\mathrm{II}}^{\mathrm{INT}} \simeq 4 s_{14} s_{24} s_{13} s_{23} \sin \Delta \sin \left(\Delta+\delta_{13}-\delta_{14}\right) .
$$

From eqs. (2.5)-(2.7), we can observe that the transition probability depends upon three small mixing angles: the standard angle $\theta_{13}$ and two new angles $\theta_{14}$ and $\theta_{24}$. We notice that the estimates of such three mixing angles (calculated in the 3-flavor framework [5860] for $\theta_{13}$, and in the 4-flavor scheme [61-64] for $\theta_{14}$ and $\theta_{24}$ ) are similar and one has $s_{13} \sim s_{14} \sim s_{24} \sim 0.15$ (see table 1). Therefore, one can consider these three angles as small parameters having the same order $\epsilon$. We note also that the ratio of the solar over the 
atmospheric mass-squared splittings, $\alpha \equiv \Delta m_{21}^{2} / \Delta m_{31}^{2} \simeq \pm 0.03$ can be treated as of order $\epsilon^{2}$. From eqs. (2.5)-(2.7), we deduce that the first (leading) contribution is of the second order, while the two interference terms are of the third one. However, differently from the standard interference term in eq. (2.6), the new sterile induced interference term in eq. (2.7) is not proportional to $\Delta$, so it is not enhanced at the second oscillation maximum. Because of this feature, as it will be confirmed by our numerical simulations, the performance of $\mathrm{ESS} \nu \mathrm{SB}$ in the $3+1$ scheme is not as good as that of those experiments which work at the first oscillation maximum, such as T2HK and DUNE.

\section{Experimental specifications}

In this section, we briefly discuss the specifications of the experimental setup ESS $\nu \mathrm{SB}$. $\mathrm{ESS} \nu \mathrm{SB}$ is a proposed superbeam on-axis experiment where a very high intense proton beam of energy $2 \mathrm{GeV}$ with an average beam power of $5 \mathrm{MW}$ will be delivered by the European Spallation Source (ESS) linac facility running at $14 \mathrm{~Hz}$. The number of protons on target (POT) per year (208 days) will be $2.7 \times 10^{23}$ [65-68]. It is worth to mention here that the future linac upgrade can push the proton energy up to $3.6 \mathrm{GeV}$. This highly ambitious and exciting facility is expected to start taking neutrino data around 2030. We have obtained the fluxes from [69] and these on-axis (anti)neutrino fluxes arising from the $2 \mathrm{GeV}$ protons on target peaks around $0.25 \mathrm{GeV}$. In this case a $500 \mathrm{kt}$ fiducial mass Water Cherenkov detector similar to the properties of the MEMPHYS detector [70, 71] has been proposed to explore the neutrino properties in this low energy regimes. It has been shown in [65] that if the detector is placed in any of the existing mines located in between $300-600 \mathrm{~km}$ from the ESS site at Lund, it will make possible to achieve $5 \sigma$ confidence level discovery of leptonic CP-violation up to the $50 \%$ coverage of the whole range of $\mathrm{CP}$ phases. A detailed study on the CP-violation discovery capability of this facility with different baseline and different combinations of neutrino and antineutrino run time has also been explored in [72]. In this work, we consider a baseline of $540 \mathrm{~km}$ from Lund to Garpenberg mine located in Sweden and also we have matched the event numbers of table 3 and all other results given in [65]. At this baseline, it fully covers the second oscillation maximum and it provides the opportunity to explore the CP-asymmetry which (in the 3-flavor scheme) is three times larger than the CP-asymmetry at the first oscillation maximum. Although the main drawbacks for going to the second oscillation maximum come from the significant decrease of statistics and cross-sections compared to the first oscillation maximum, the high intense beam of this excellent facility takes care of those difficulties and make the statistics competitive to provide exciting results. All our simulations presented here for this setup have been done assuming 2 yrs of $\nu$ and 8 yrs of $\bar{\nu}$ running with a most optimistic consideration of uncorrelated $5 \%$ signal normalization and $10 \%$ background normalization error for both neutrino and antineutrino appearance and disappearance channels respectively. For more details of the accelerator facility, beamline design, and detector facility of this setup please see [65]. 


\begin{tabular}{|c|c|c|}
\hline Parameter & True Value & Marginalization Range \\
\hline $\sin ^{2} \theta_{12}$ & 0.304 & Not marginalized \\
\hline $\sin ^{2} 2 \theta_{13}$ & 0.085 & Not marginalized \\
\hline $\sin ^{2} \theta_{23}$ & 0.50 & {$[0.34,0.68]$} \\
\hline $\sin ^{2} \theta_{14}$ & 0.025 & Not marginalized \\
\hline $\sin ^{2} \theta_{24}$ & 0.025 & Not marginalized \\
\hline $\sin ^{2} \theta_{34}$ & 0.0 & Not marginalized \\
\hline$\delta_{13} /^{\circ}$ & {$[-180,180]$} & {$[-180,180]$} \\
\hline$\delta_{14} /^{\circ}$ & {$[-180,180]$} & {$[-180,180]$} \\
\hline$\delta_{34} /^{\circ}$ & 0 & Not marginalized \\
\hline$\frac{\Delta m_{21}^{2}}{10^{-5} \mathrm{eV}^{2}}$ & 7.50 & Not marginalized \\
\hline$\frac{\Delta m_{31}^{2}}{10^{-3} \mathrm{eV}^{2}}(\mathrm{NH})$ & 2.475 & Not marginalized \\
\hline$\frac{\Delta m_{31}^{2}}{10^{-3} \mathrm{eV}^{2}}(\mathrm{IH})$ & -2.4 & Not marginalized \\
\hline$\frac{\Delta m_{41}^{2}}{\mathrm{eV}^{2}}$ & 1.0 & Not marginalized \\
\hline
\end{tabular}

Table 1. Oscillation parameters along with their true values and marginalization status shown in this table. The second column represents the values of the parameters used to generate the true data set. The third column displays the parameters which are kept fixed in the fit and the parameters which have been marginalized in the fit within their allowed ranges.

\section{Details of the numerical analysis}

This section details the numerical analysis adopted to produce the sensitivity results presented in the following sections. To compute the sensitivity measurements along with the 
bi-events plots we have used the GLoBES software [73, 74] and its new tool [75] which can include the sterile neutrinos. In this paper, we have adopted the same strategy for the simulation described in section 4 of ref. [76]. The true values of the oscillation parameters together with their marginalization ranges considered in our simulations are presented in table 1. Our benchmark choices for the three-flavor neutrino oscillation parameters closely resemble those obtained in the latest global fits [58-60], although we have made the true choice of the atmospheric mixing angle to be maximal $\left(45^{\circ}\right),{ }^{2}$ and in the fit, it has been marginalized over its allowed range as mentioned in the third column of table 1 . Concerning the active-sterile mixing angles we have taken the benchmark values very close to those obtained in the global fit analyses [61-64] performed within the $3+1$ scheme. $^{3}$

In all our simulations, we have assumed normal hierarchy ${ }^{4}(\mathrm{NH})$ as the true choice and we have kept it fixed also in the fit. In fact, we are assuming that the correct hierarchy will be already known by the time $\mathrm{ESS} \nu \mathrm{SB}$ will start to take data. The two mixing angles $\theta_{12}$ and $\theta_{13}$ have been kept fixed in the data as well as in the fit taking into account the stringent constraints provided by the solar and the reactor data. We have also kept the two mass-squared differences $\Delta m_{21}^{2}$ and $\Delta m_{31}^{2}$ fixed at their true choices and they have not been marginalized in the fit. $\delta_{13}$ (true) has been taken from its allowed range of $[-\pi, \pi]$, while in the fit we have marginalized over its full range depending on the analysis requirement. In our simulations, we consider the constant line-averaged Earth matter density of $2.8 \mathrm{~g} / \mathrm{cm}^{3}$ following the Preliminary Reference Earth Model (PREM) [77]. The new mass-squared splitting $\Delta m_{41}^{2}$ arising in the $3+1$ scheme is taken as $1 \mathrm{eV}^{2}$ following the present preference of the short-baseline data. ${ }^{5}$ This large value of $\Delta m_{41}^{2}$ induces fast oscillations which get averaged out due to the finite energy resolution of the detector. As a result the sign of $\Delta m_{41}^{2}$ is irrelevant in this setup. Now, the new mixing angles $\theta_{14}$ and $\theta_{24}$ emerging out of the $3+1$ framework, have been taken fixed at their true values in the data as well as in the fit. ${ }^{6}$

The true value of the new $\mathrm{CP}$ phase $\delta_{14}$ is taken in its allowed range $[-\pi, \pi]$ and its test value has been marginalized over the allowed range if required. The mixing angle $\theta_{34}$ has been considered to be zero both in the data and in theory. This choice makes the presence of its associated phase $\delta_{34}$ irrelevant in the simulation. ${ }^{7}$

\footnotetext{
${ }^{2}$ Recent $3 \nu$ global fits [58-60] sligthy prefer non-maximal $\theta_{23}$ with two nearly degenerate solutions: one is $<45^{\circ}$, in the lower octant (LO), and the other is $>45^{\circ}$, in the higher octant (HO). However, maximal mixing is still allowed at $2 \sigma$ confidence level.

${ }^{3}$ We stress that assuming smaller values for $\theta_{14}$ and $\theta_{24}$ the impact of active-sterile oscillations would decrease. As a consequence the sensitivity to CPV induced by $\delta_{14}$ would be reduced. On the other hand, the deterioration of the sensitivity to the CPV induced by the standard CP phase $\delta_{13}$ would be less.

${ }^{4}$ We have checked that the results with the true choice of inverted hierarchy are similar to the results presented in this work.

${ }^{5}$ We stress that our sensitivity results would remain unaltered provided $\Delta m_{41}^{2} \gtrsim 0.1 \mathrm{eV}^{2}$.

${ }^{6}$ We point out that our choice to fix the fit values of $\theta_{14}$ and $\theta_{24}$ is well justified if one assumes (as we do) that one has precise information on these two parameters coming form SBL experiments. In such a case, the marginalization of $\theta_{14}$ and $\theta_{24}$ in the fit would provide minor modifications to our results.

${ }^{7}$ According to our parametrization followed in eq. (2.1), the $\nu_{\mu} \rightarrow \nu_{e}$ oscillation probability in vacuum is independent of $\theta_{34}$ (and $\delta_{34}$ ). However it has a higher order $\left(\epsilon^{4}\right)$ impact in presence of matter effect, which in case of $\operatorname{ESS} \nu \mathrm{SB}$ baseline is very small. Hence $\theta_{34}$ (and $\delta_{34}$ ) can safely be ignored in the simulation. A detailed discussion including some analytical understanding regarding this issue is given in the appendix of ref. [27].
} 

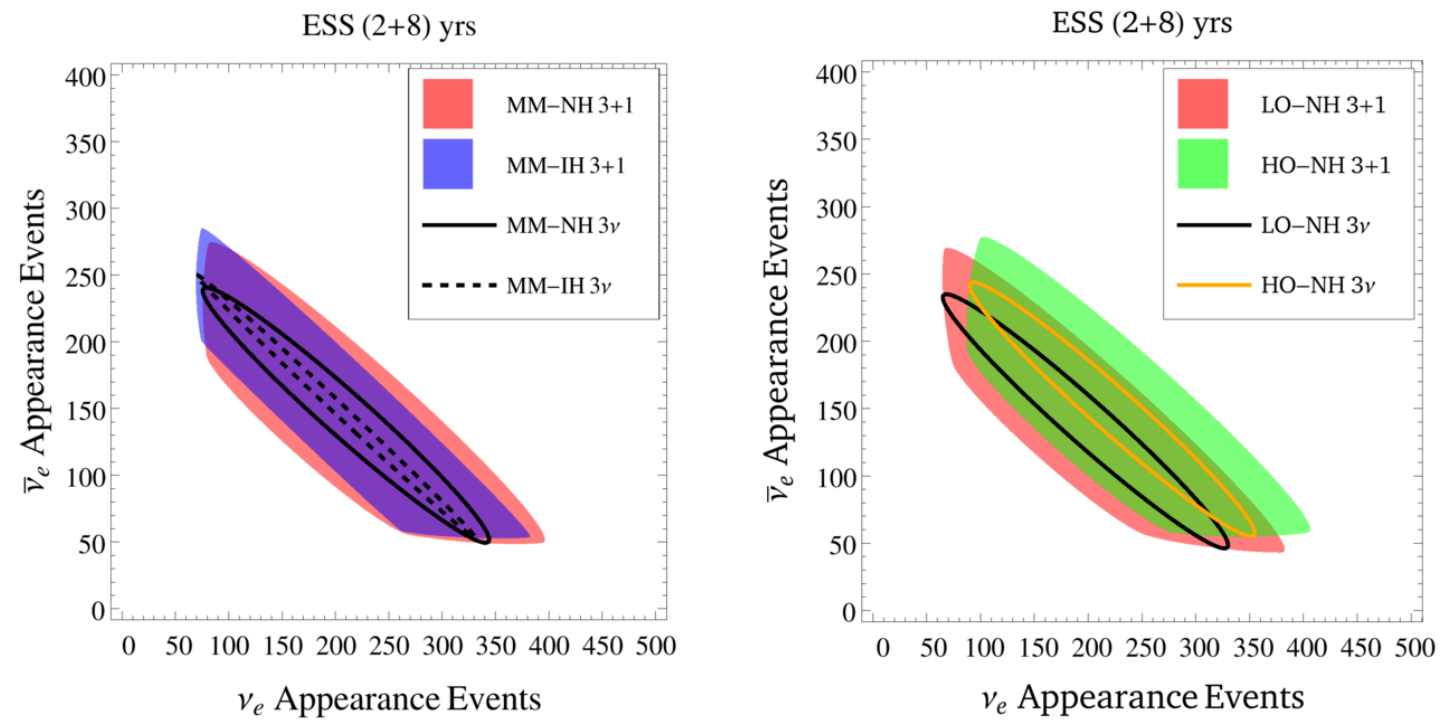

Figure 1. The left panel reports the bievents plot for the 3-flavor framework (black curves) and $3+1$ scheme (colored blobs) for the case of $\mathrm{NH}$ and maximal $\theta_{23}$. The right panel represents, for the NH case, the bievents plots corresponding to two values of $\theta_{23}$ in the two octants. We have taken $\sin ^{2} \theta_{23}=0.42(0.58)$. See the text for details.

In our analysis, we do not consider any near detector of $\mathrm{ESS} \nu \mathrm{SB}$ which may help to reduce the systematic uncertainties and might give some information on the two mixing angles $\theta_{14}$ and $\theta_{24}$. However, it would give no information regarding the active and sterile CP phases which is our main issue of interest in the present work. It is worth to underline here that in all our simulations we have performed a spectral analysis making use of the binned events spectra. In the statistical analysis we not only marginalize over the oscillation parameters but also over the nuisance parameters adopting the well-known "pull" method $[78,79]$ to calculate the Poissonian $\Delta \chi^{2}$. We display our results in terms of the squared-root of $\Delta \chi^{2}$ which represents $n \sigma\left(n \equiv \sqrt{\Delta \chi^{2}}\right)$ confidence level statistical significance for one degrees of freedom (d.o.f).

\section{Mass ordering and $\theta_{23}$ octant sensitivity in the 4-flavor scheme}

It is well known that in the 3-flavor scheme $\operatorname{ESS} \nu \mathrm{SB}$ has scarce sensitivity to both these two properties. Concerning the MH hierarchy, the lack of sensitivity is due to the fact that matter effects are very small in $\mathrm{ESS} \nu \mathrm{SB}$. The low sensitivity to the octant of $\theta_{23}$ is imputable to the fact that $\mathrm{ESS} \nu \mathrm{SB}$ works at the second oscillation maximum, which is more narrow than the first one. These features, together with the lower statistics, render ESS $\nu \mathrm{SB}$ much less sensitive than other experiments (T2HK for example) to the octant of $\theta_{23}$.

Here we confirm similar findings also in the 4 -flavor scheme. This conclusion can be easily understood through a discussion at the level of the neutrino and antineutrino appearance events. The left panel of figure 1 reports the bievent plots, where the $x$-axis represents the number of $\nu_{e}$ events and the $y$-axis represents the $\bar{\nu}_{e}$ events. The two ellipses represent the 
3 -flavor model and can be obtained by varying the CP phase $\delta_{13}$ in the range $[-\pi, \pi]$. The solid (dashed) ellipse corresponds to the $\mathrm{NH}(\mathrm{IH})$. The centroids of the two ellipses basically coincide, hence it is clear that the setup cannot discriminate the MH. This is qualitatively different with respect to what occurs in other LBL experiments (T2HK [46] and especially DUNE [41]), where the two ellipses get separated due to the presence of the matter effects. In the $3+1$ scheme, two $\mathrm{CP}$ phases are present and their variation in the range $[-\pi, \pi]$ gives even more freedom. The bievent plots obtained varying both the CP phases $\delta_{13}$ and $\delta_{14}$ are represented by colored elongated blobs in the left panel figure 1. The two blobs corresponding to the two hierarchies are completely overlapped. This implies that, similarly to the 3 -flavor scheme, one does not expect any sensitivity to the $\mathrm{MH}$ in the $3+1$ scheme as well.

The right panel of figure 1 reports the ellipses (blobs) obtained in the 3 -flavor (4flavor) cases for two values of $\theta_{23}$ chosen in the two opposite octants. We have taken $\sin ^{2} \theta_{23}=0.42(0.58)$ as benchmark values. We observe that in both schemes there is a partial overlapping between the regions representing the two octants. The degree of overlapping increases when going from 3 -flavor to the $3+1$ scheme. Therefore, we expect a poor sensitivity to the octant of $\theta_{23}$ both in $3 \nu$ and $4 \nu$ schemes. Differently from T2HK, the spectral information is not of great help due to the low statistics. This is confirmed by the numerical simulations (not shown) performed by including the full energy spectrum in the fit.

\section{CP-violation searches in the 4-flavor framework}

In this section, we analyze the capability of $\mathrm{ESS} \nu \mathrm{SB}$ of pinning down the extended CPV sector entailed by the $3+1$ scheme. First we assess the sensitivity to the CPV induced by the CP phase $\delta_{13}$ and $\delta_{14}$. Second we discuss the capability of reconstructing the true values of the two phases $\delta_{13}$ and $\delta_{14}$.

\subsection{Sensitivity to CP-violation}

The sensitivity of CPV produced by a fixed (true) value of a CP phase $\delta_{i j}^{\text {true }}$ can be defined as the statistical significance at which one can reject the test hypothesis of no CPV, i.e. the two (test) cases $\delta_{i j}^{\text {test }}=0, \pi$. In the left panel of figure 2, we report the discovery potential of CPV induced by $\delta_{13}$. We have assumed that the hierarchy is known a priori and is NH. The dashed black curve correspond to the 3 -flavor scheme while the green band to the $3+1$ scheme. In the $3+1$ scenario, we fix the test and true values of $\theta_{14}=9^{0}$ and $\theta_{24}=9^{0}$. The green band is attained by varying the unknown true value of $\delta_{14}$ in the range of $[-\pi, \pi]$ and marginalizing over its test values. We observe that in the $3+1$ scheme there is a deterioration of the sensitivity. Adopting $\delta_{13}=-90^{0}$ as a benchmark value in the 3 -flavor (4-flavor) scheme one has $8.2 \sigma(4.5 \sigma)$ sensitivity. We find very similar result for the case of IH (not shown). The right panel of figure 2 displays the discovery potential of CPV induced by $\delta_{14}$ for the NH case. The magenta band is obtained by varying the true values of the $\mathrm{CP}$ phase $\delta_{13}$ in the range $[-\pi, \pi]$ while marginalizing over their test values in the same range in the fit. We observe that $\operatorname{ESS} \nu \mathrm{SB}$ has a limited sensitivity to the $\mathrm{CP}$ phase $\delta_{14}$, which is always below the $2 \sigma$ level. 

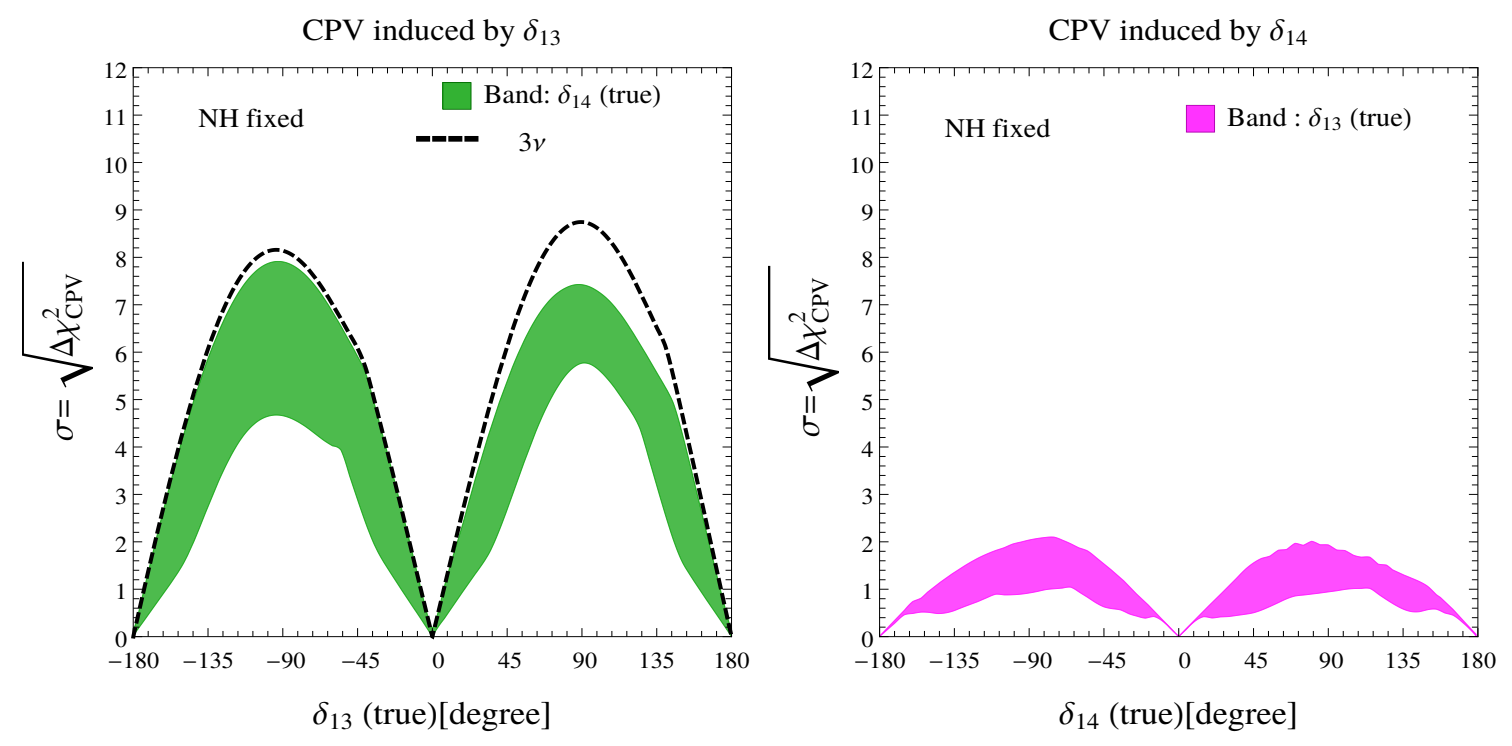

Figure 2. $\mathrm{ESS} \nu \mathrm{SB}$ discovery potential of $\delta_{13} \neq(0, \pi)$ (left panel) and $\delta_{14} \neq(0, \pi)$ (right panel). In both panels the $\mathrm{MH}$ is fixed to be the $\mathrm{NH}$ (both true and test value). The black dashed curve corresponds to the 3 -flavor case while the colored band correspond to the $3+1$ scheme. In this last case, we have fixed the true and test values of $\theta_{14}=\theta_{24}=9^{0}$ and varied the unknown value of the true $\delta_{14}$ in its entire range of $[-\pi, \pi]$ while marginalizing over test $\delta_{14}$ in the same range.

We think that it is useful to make a comparison between the results obtained here for $\mathrm{ESS} \nu \mathrm{SB}$ with those found for T2HK in our work [46]. We notice that in the 3-flavor scheme both experiments have a similar sensitivity to CPV induced by $\delta_{13}$, having both a maximal sensitivity of about $8 \sigma$ for the values $\delta_{13} \simeq \pm 90^{0}$. This is possible because, despite of the lower statical power, $\mathrm{ESS} \nu \mathrm{SB}$ benefits of the amplification factor proportional to $\Delta$, which is three times bigger at the second oscillation maximum with respect to the first one. In contrast, in the presence of a sterile neutrino, the performance is much worse in $\mathrm{ESS} \nu \mathrm{SB}$. In fact, one can notice the two following features: i) The deterioration of the sensitivity to the CPV driven by $\delta_{13}$ when going from the 3 -flavor to the $3+1$ scheme is much more pronounced in $\mathrm{ESS} \nu \mathrm{SB}$ than in T2HK. Taking the values $\delta_{13}= \pm 90^{0}$ as a benchmark (where the maximal sensitivity is attained) in [46], we found for T2HK only a weak reduction of the sensitivity from $8 \sigma$ to $7 \sigma$ (see figure 4 in [46]). In $\mathrm{ESS} \nu \mathrm{SB}$, we now find a severe reduction from $8 \sigma$ to $4.5 \sigma$ (see left panel of figure 2); ii) The sensitivity to the CPV induced by the $\mathrm{CP}$ phase $\delta_{14}$ is considerably lower in $\mathrm{ESS} \nu \mathrm{SB}$ than in T2HK ( $2 \sigma$ vs $5 \sigma$ for $\delta_{14}= \pm 90^{0}$ ). The explanation of such a different performance in the $3+1$ scheme of the two experiments can be traced to the fact that T2HK (ESS $\nu \mathrm{SB}$ ) works around the first (second) oscillation maximum. As already noticed in subection 2.2, the new interference term (which depends on $\delta_{14}$ ), at the second oscillation maximum is not amplified by the factor $\Delta$ as it happens for the standard interference term (which depends on $\delta_{13}$ ). In addition, as remarked in [46] in T2HK the spectral information plays a crucial role in guaranteeing a good performance in the $3+1$ scheme. Indeed, in [46], we explicitly showed that even if there is a complete degeneracy at the level of the event counting, the energy spectrum provides additional 

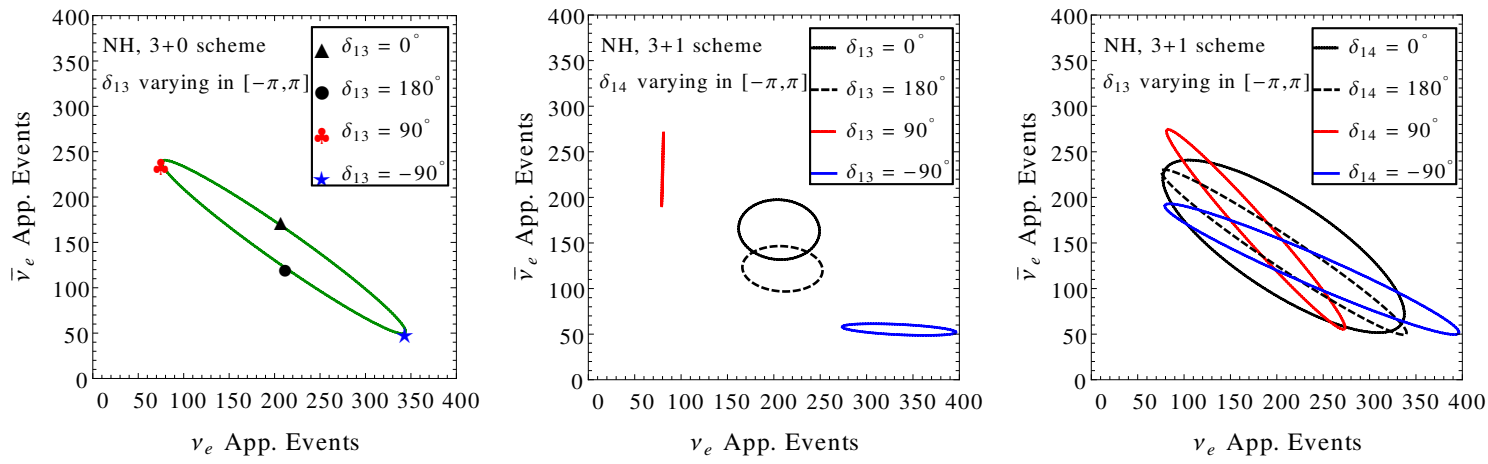

Figure 3. The left panel refers to the standard 3-flavor framework. In this case the model lies on the (green) ellipse, which is obtained by varying $\delta_{13}$ in the range $[-\pi, \pi]$. The two black marks represent the cases of no CPV $\left(\delta_{13}=0, \pi\right)$ while the two colored ones correspond to the cases of maximal CPV $\left(\delta_{13}=-\pi / 2, \pi / 2\right)$. The non-zero distance between the black marks and the colored ones implies that events counting can detect the CPV induced by the phase $\delta_{13}$. The second panel refers to the $3+1$ scheme. In this case a fixed value of $\delta_{13}$ is represented by an ellipse, where $\delta_{14}$ varies in the range $[-\pi, \pi]$. The non-zero distance between the black ellipses and the two colored ones implies that events counting is sensitive to CPV induced by the phase $\delta_{13}$ also in the $3+1$ case. However, the distances are reduced with respect to the 3 -flavor case. Therefore, the sensitivity decreases. The right panel refers to the $3+1$ scheme and illustrates the sensitivity to the CPV induced by $\delta_{14}$. In this case we plot four ellipses corresponding to the four values of $\delta_{14}$ (while $\delta_{13}$ is varying in the range $[-\pi, \pi]$ ). Each of the two ellipses (blue and red) corresponding to maximal CPV induced by $\delta_{14}$ intercepts the two ellipses (solid and dashed black) corresponding to no CPV induced by $\delta_{14}$. In the crossing points the events counting is completely insensitive to CPV induced by the new CP phase $\delta_{14}$.

information which breaks such a degeneracy and boosts the sensitivity. In ESS $\nu \mathrm{SB}$, the role of the spectral information is substantially reduced because of the energy range at the second oscillation maximum is very narrow and the low statistics does not allow to exploit the information contained in the spectrum. Hence, we conclude that ESS $\nu \mathrm{SB}$ is not particularly suited for the CPV related searches in the presence of sterile neutrinos.

The situation can be further clarified by inspecting the 3-panel bievent plot displayed in figure 3. The left panel refers to the standard 3-flavor framework. In this case the model lies on the (green) ellipse, which is obtained by varying $\delta_{13}$ in the range $[-\pi, \pi]$. The two black marks represent the cases of no $\operatorname{CPV}\left(\delta_{13}=0, \pi\right)$ while the two colored ones correspond to the cases of maximal CPV $\left(\delta_{13}=-\pi / 2, \pi / 2\right)$. The non-zero distance between the black marks and the colored ones implies that events counting can detect the CPV induced by the phase $\delta_{13}$. The second panel refers to the $3+1$ scheme. In this case a fixed value of $\delta_{13}$ is represented by an ellipse, where $\delta_{14}$ varies in the range $[-\pi, \pi]$. The non-zero distance between the black ellipses and the two colored ones implies that events counting is sensitive to $\mathrm{CPV}$ induced by the phase $\delta_{13}$ also in the $3+1$ case. However, the distances are reduced with respect to the 3 -flavor case. Therefore, the sensitivity decreases as found in the numerical simulation as shown in the left panel of figure 2 . The right panel of figure 3 refers to the $3+1$ scheme and illustrates the sensitivity to the CPV induced by $\delta_{14}$. In this case we plot four ellipses corresponding to the four values of $\delta_{14}$ (while $\delta_{13}$ is varying 

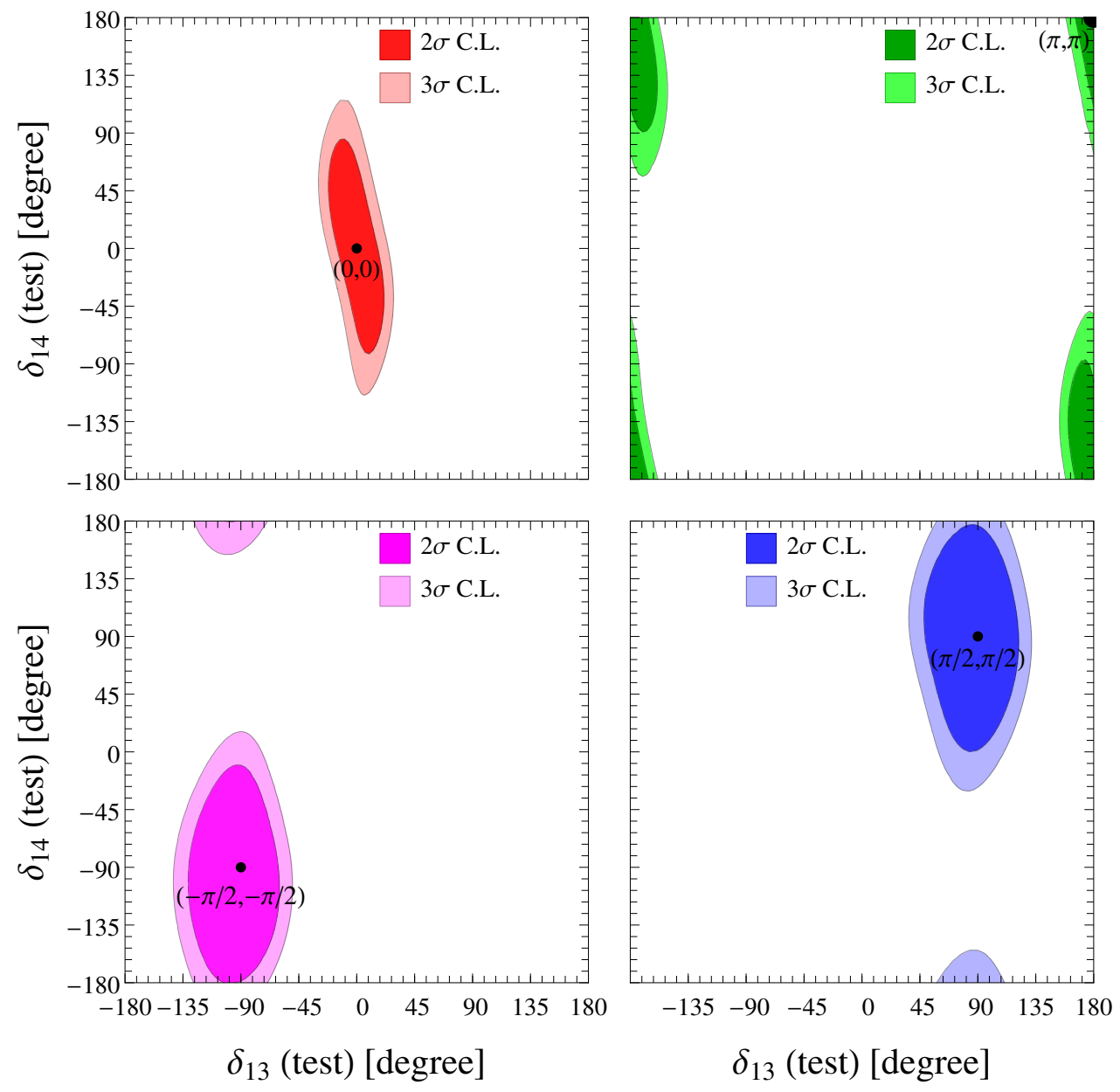

Figure 4. Reconstructed regions for the two CP phases $\delta_{13}$ and $\delta_{14}$ for the four benchmark pairs of their true values indicated in each panel. We have fixed the NH as the true and test hierarchy. The contours refer to $2 \sigma$ and $3 \sigma$ confidence levels (1 d.o.f.).

in the range $[-\pi, \pi]$ ). Each of the two ellipses (blue and red) corresponding to maximal CPV induced by $\delta_{14}$ intercepts the two ellipses (solid and dashed black) corresponding to no CPV induced by $\delta_{14}$. In the crossing points the events counting is completely insensitive to CPV induced by the new CP phase $\delta_{14}$. Therefore there are always (unlucky) combinations of the CP phases for which the event counting cannot determine if there is CPV induced by $\delta_{14}$. Notwithstanding in the right panel of figure 2 , we observe that there is $\sim 2 \sigma$ sensitivity for $\delta_{14}= \pm 90^{\circ}$. We have checked that such a residual sensitivity comes from the spectral shape information. As already remarked above, this information in ESS $\nu \mathrm{SB}$ is much weaker compared to T2HK, and as a consequence the sensitivity remains quite low. 

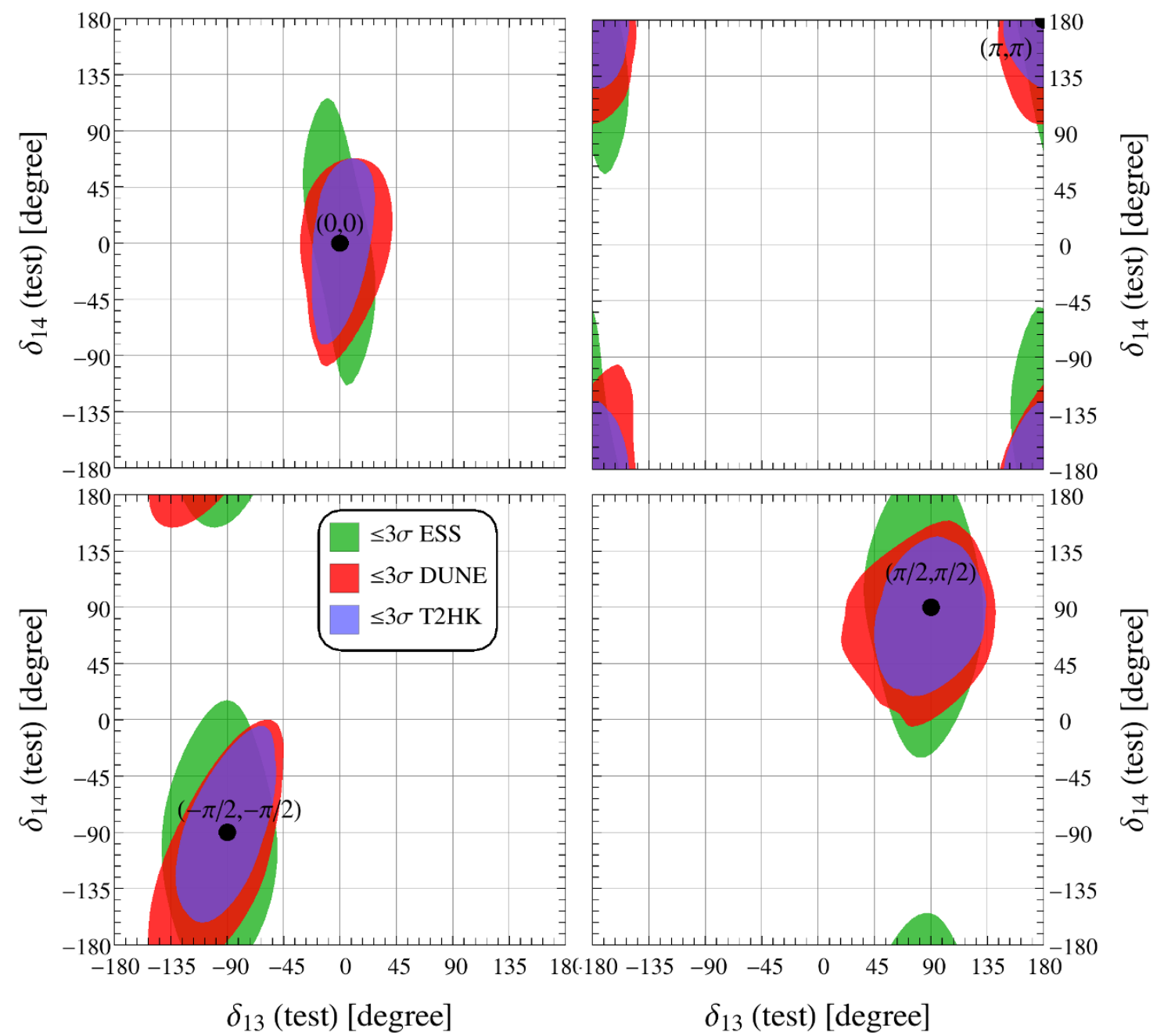

Figure 5. Reconstructed regions for the two CP phases $\delta_{13}$ and $\delta_{14}$ for the four benchmark pairs of their true values indicated in each panel. Results are shown for the three different experimental setups: ESS $\nu \mathrm{SB}, \mathrm{DUNE}$, and T2HK. We have fixed the NH as the true and test hierarchy. The contours correspond to $3 \sigma$ (1 d.o.f.) confidence level.

\subsection{Reconstructing the CP phases}

So far we have discussed the sensitivity to the CPV induced by the two CP phases $\delta_{13}$ and $\delta_{14}$. Here, we study the ability of the $\mathrm{ESS} \nu \mathrm{SB}$ setup to reconstruct the two CP phases. With this aim, we focus on the four benchmark cases shown in figure 4 . The first two panels correspond to the CP-conserving cases $(0,0)$ and $(\pi, \pi)$. The lower panels represent two CP-violating scenarios $(-\pi / 2,-\pi / 2)$ and $(\pi / 2, \pi / 2)$. In each panel, we show the regions reconstructed close to the true values of the two $\mathrm{CP}$ phases. In this figure we have fixed the $\mathrm{NH}$ as the true and test hierarchy. The contours are shown for the two different confidence 
levels: $2 \sigma$ and $3 \sigma$ (1 d.o.f.). The typical $1 \sigma$ level uncertainty on the reconstructed $\mathrm{CP}$ phases is approximately $15^{0}\left(35^{0}\right)$ for $\delta_{13}\left(\delta_{14}\right) .8$

We end this section by comparing the performance of the $\mathrm{ESS} \nu \mathrm{SB}$ setup with the two other proposed long-baseline facilities: $\mathrm{DUNE}^{9}$ and T2HK. ${ }^{10}$ In figure 5 , we show the reconstructed regions for $\mathrm{ESS} \nu \mathrm{SB}, \mathrm{DUNE}$, and T2HK for the same benchmark values of the true phases considered in figure 4 . To have the visual clearness, we only depict the $3 \sigma$ (1 d.o.f.) contours. While making this plot, we consider the $\mathrm{NH}$ as the true and test hierarchy. The performance of $\mathrm{ESS} \nu \mathrm{SB}$ in reconstructing $\delta_{13}$ is almost similar to that of DUNE and T2HK. In contrast, the reconstruction of $\delta_{14}$ is slightly better for T2HK and DUNE as compared to $\mathrm{ESS} \nu \mathrm{SB}$.

\section{Conclusions and outlook}

We have studied in detail the potential of $\mathrm{ESS} \nu \mathrm{SB}$ in the presence of a light eV-scale sterile neutrino with an emphasis on the CPV searches. We have presented our results assuming a baseline of $540 \mathrm{~km}$, which provides a platform to exploit the featutres of the second oscillation maximum. We have found that the sensitivity to CPV driven by the standard CP phase $\delta_{13}$ substantially deteriorates with respect to the standard 3-flavor case. More specifically, the maximal sensitivity (assumed for $\delta_{13} \sim \pm 90^{\circ}$ ) drops from $8 \sigma$ down to $4.5 \sigma$ if the size of the mixing angles $\theta_{14}$ and $\theta_{24}$ is similar to that of $\theta_{13}$. The sensitivity to the CPV induced by $\delta_{14}$ is modest and never exceeds the $2 \sigma$ level for the baseline choice of 540 $\mathrm{km}$. We have also studied the ability of reconstructing the two phases $\delta_{13}$ and $\delta_{14}$. The $1 \sigma$ error on $\delta_{13}\left(\delta_{14}\right)$ is $\sim 15^{0}\left(35^{0}\right)$. As far as the octant of $\theta_{23}$ is concerned, the benchmark setup under consideration for the $\mathrm{ESS} \nu \mathrm{SB}$ experiment provides poor results in the 3 -flavor scenario and performs even worse in $3+1$ scheme. Needless to mention that ESS $\nu$ SB benefits a lot from working at the second oscillation maximum and provides excellent sensitivity to $\mathrm{CPV}$ in $3 \nu$ scheme. However, in the present work we find that this setup with a baseline of $540 \mathrm{~km}$ is not optimal for exploring fundamental neutrino properties in $3+1$ scenario.

\section{Acknowledgments}

S.K.A. is supported by the DST/INSPIRE Research Grant [IFA-PH-12] from the Department of Science and Technology (DST), India and the Indian National Science Academy (INSA) Young Scientist Project [INSA/SP/YSP/144/2017/1578]. S.K.A. would like to thank Tord Ekelof for useful discussions. S.S.C. acknowledges the partial support from the DST/INSPIRE Research Grant [IFA-PH-12], Department of Science and Technology, India during the initial stage of this project at Institute of Physics (IOP), Bhubaneswar, India. This work made extensive use of the cluster facilities at IOP, Bhubaneswar, India. A.P.

\footnotetext{
${ }^{8}$ Note that both $\delta_{13}$ and $\delta_{14}$ are cyclic variables, hence, the four corners in the top right panel of figure 4 give rise to a unique connected region.

${ }^{9}$ To simulate the DUNE setup, we consider the reference design as mentioned in the Conceptual Design Report (CDR) [34] and the necessary simulation files for the GLoBES software are taken from [80].

${ }^{10}$ To estimate the reconstruction capability of the T2HK setup, we closely follow the experimental configurations as described in refs. [32, 81].
} 
acknowledges partial support by the research grant number 2017W4HA7S "NAT-NET: Neutrino and Astroparticle Theory Network" under the program PRIN 2017 funded by the Italian Ministero dell'Istruzione, dell'Università e della Ricerca (MIUR) and by the research project TAsP funded by the Instituto Nazionale di Fisica Nucleare (INFN).

Open Access. This article is distributed under the terms of the Creative Commons Attribution License (CC-BY 4.0), which permits any use, distribution and reproduction in any medium, provided the original author(s) and source are credited.

\section{References}

[1] K.N. Abazajian et al., Light sterile neutrinos: a white paper, arXiv:1204.5379 [INSPIRE].

[2] A. Palazzo, Phenomenology of light sterile neutrinos: a brief review, Mod. Phys. Lett. A 28 (2013) 1330004 [arXiv: 1302.1102] [INSPIRE].

[3] S. Gariazzo, C. Giunti, M. Laveder, Y.F. Li and E.M. Zavanin, Light sterile neutrinos, J. Phys. G 43 (2016) 033001 [arXiv: 1507.08204] [INSPIRE].

[4] C. Giunti, Light sterile neutrinos: status and perspectives, Nucl. Phys. B 908 (2016) 336 [arXiv: 1512.04758] [INSPIRE].

[5] C. Giunti and T. Lasserre, eV-scale sterile neutrinos, arXiv:1901.08330 [INSPIRE].

[6] S. Böser et al., Status of light sterile neutrino searches, arXiv:1906.01739 [INSPIRE].

[7] LSND collaboration, Evidence for neutrino oscillations from the observation of $\bar{\nu}_{e}$ appearance in a $\bar{\nu}_{\mu}$ beam, Phys. Rev. D 64 (2001) 112007 [hep-ex/0104049] [INSPIRE].

[8] MiniBoone collaboration, Significant excess of electronlike events in the MiniBooNE short-baseline neutrino experiment, Phys. Rev. Lett. 121 (2018) 221801 [arXiv:1805.12028] [INSPIRE].

[9] G. Mention et al., The reactor antineutrino anomaly, Phys. Rev. D 83 (2011) 073006 [arXiv:1101.2755] [INSPIRE].

[10] GALLEX collaboration, Final results of the ${ }^{51}$ Cr neutrino source experiments in GALLEX, Phys. Lett. B 420 (1998) 114 [INSPIRE].

[11] J.N. Abdurashitov et al., Measurement of the response of a Ga solar neutrino experiment to neutrinos from an ${ }^{37}$ Ar source, Phys. Rev. C 73 (2006) 045805 [nucl-ex/0512041] [INSPIRE].

[12] MINOS collaboration, Search for sterile neutrinos mixing with muon neutrinos in MINOS, Phys. Rev. Lett. 117 (2016) 151803 [arXiv:1607.01176] [INSPIRE].

[13] MINOS+ collaboration, Search for sterile neutrinos in MINOS and MINOS+ using a two-detector fit, Phys. Rev. Lett. 122 (2019) 091803 [arXiv:1710.06488] [INSPIRE].

[14] NOvA collaboration, Search for active-sterile neutrino mixing using neutral-current interactions in NOvA, Phys. Rev. D 96 (2017) 072006 [arXiv: 1706.04592] [INSPIRE].

[15] T2K collaboration, Search for light sterile neutrinos with the T2K far detector Super-Kamiokande at a baseline of 295 km, Phys. Rev. D 99 (2019) 071103 [arXiv: 1902.06529] [INSPIRE]. 
[16] DAYA BAY collaboration, Improved search for a light sterile neutrino with the full configuration of the Daya Bay experiment, Phys. Rev. Lett. 117 (2016) 151802 [arXiv: 1607.01174] [INSPIRE].

[17] DAYA BAY and MINOS collaborations, Limits on active to sterile neutrino oscillations from disappearance searches in the MINOS, Daya Bay and Bugey-3 experiments, Phys. Rev. Lett. 117 (2016) 151801 [Addendum ibid. 117 (2016) 209901] [arXiv:1607.01177] [INSPIRE].

[18] DANSS collaboration, Recent results of the DANSS experiment, in 2019 European Physical Society Conference on High Energy Physics (EPS-HEP2019), Ghent, Belgium, 10-17 July 2019 [arXiv: 1911.10140] [INSPIRE].

[19] NEOS collaboration, Sterile neutrino search at the NEOS experiment, Phys. Rev. Lett. 118 (2017) 121802 [arXiv: 1610.05134] [INSPIRE].

[20] SuPER-KAMIOKANDE collaboration, Limits on sterile neutrino mixing using atmospheric neutrinos in Super-Kamiokande, Phys. Rev. D 91 (2015) 052019 [arXiv:1410.2008] [INSPIRE].

[21] ICECUBE collaboration, Search for sterile neutrino mixing using three years of IceCube DeepCore data, Phys. Rev. D 95 (2017) 112002 [arXiv:1702.05160] [INSPIRE].

[22] ANTARES collaboration, Measuring the atmospheric neutrino oscillation parameters and constraining the $3+1$ neutrino model with ten years of ANTARES data, JHEP 06 (2019) 113 [arXiv:1812.08650] [INSPIRE].

[23] C. Giunti and Y.F. Li, Matter effects in active-sterile solar neutrino oscillations, Phys. Rev. D 80 (2009) 113007 [arXiv:0910.5856] [INSPIRE].

[24] A. Palazzo, Testing the very-short-baseline neutrino anomalies at the solar sector, Phys. Rev. D 83 (2011) 113013 [arXiv:1105.1705] [INSPIRE].

[25] A. Palazzo, An estimate of $\theta_{14}$ independent of the reactor antineutrino flux determinations, Phys. Rev. D 85 (2012) 077301 [arXiv:1201.4280] [INSPIRE].

[26] T. Lasserre, Light sterile neutrinos in particle physics: experimental status, Phys. Dark Univ. 4 (2014) 81 [arXiv: 1404.7352] [INSPIRE].

[27] N. Klop and A. Palazzo, Imprints of CP-violation induced by sterile neutrinos in T2K data, Phys. Rev. D 91 (2015) 073017 [arXiv:1412.7524] [InSPIRE].

[28] G.J. Feldman, J. Hartnell and T. Kobayashi, Long-baseline neutrino oscillation experiments, Adv. High Energy Phys. 2013 (2013) 475749 [arXiv:1210.1778] [InSPIRE].

[29] S. Pascoli and T. Schwetz, Prospects for neutrino oscillation physics, Adv. High Energy Phys. 2013 (2013) 503401 [INSPIRE].

[30] S.K. Agarwalla, S. Prakash and S. Uma Sankar, Exploring the three flavor effects with future superbeams using liquid argon detectors, JHEP 03 (2014) 087 [arXiv: 1304.3251] [INSPIRE].

[31] S.K. Agarwalla, Physics potential of long-baseline experiments, Adv. High Energy Phys. 2014 (2014) 457803 [arXiv:1401.4705] [INSPIRE].

[32] Hyper-Kamiokande Proto-Collaboration collaboration, Physics potential of a long-baseline neutrino oscillation experiment using a J-PARC neutrino beam and Hyper-Kamiokande, PTEP 2015 (2015) 053C02 [arXiv:1502.05199] [INSPIRE].

[33] L. Stanco, A view of neutrino studies with the next generation facilities, Rev. Phys. 1 (2016) 90 [arXiv: 1511.09409] [INSPIRE]. 
[34] DUNE collaboration, Long-Baseline Neutrino Facility (LBNF) and Deep Underground Neutrino Experiment (DUNE), arXiv:1512.06148 [INSPIRE].

[35] Hyper-Kamiokande collaboration, Physics potentials with the second Hyper-Kamiokande detector in Korea, PTEP 2018 (2018) 063C01 [arXiv:1611.06118] [INSPIRE].

[36] S.K. Agarwalla, M. Ghosh and S.K. Raut, A hybrid setup for fundamental unknowns in neutrino oscillations using T2HK $(\nu)$ and $\mu$-DAR $(\bar{\nu})$, JHEP 05 (2017) 115 [arXiv: 1704.06116] [INSPIRE].

[37] DUNE collaboration, The DUNE far detector interim design report volume 1: physics, technology and strategies, arXiv:1807.10334 [INSPIRE].

[38] D. Hollander and I. Mocioiu, Minimal $3+2$ sterile neutrino model at LBNE, Phys. Rev. D 91 (2015) 013002 [arXiv: 1408.1749] [INSPIRE].

[39] J.M. Berryman, A. de Gouvêa, K.J. Kelly and A. Kobach, Sterile neutrino at the Deep Underground Neutrino Experiment, Phys. Rev. D 92 (2015) 073012 [arXiv:1507.03986] [INSPIRE].

[40] R. Gandhi, B. Kayser, M. Masud and S. Prakash, The impact of sterile neutrinos on CP measurements at long baselines, JHEP 11 (2015) 039 [arXiv:1508.06275] [INSPIRE].

[41] S.K. Agarwalla, S.S. Chatterjee and A. Palazzo, Physics reach of DUNE with a light sterile neutrino, JHEP 09 (2016) 016 [arXiv:1603.03759] [INSPIRE].

[42] S.K. Agarwalla, S.S. Chatterjee and A. Palazzo, Octant of $\theta_{23}$ in danger with a light sterile neutrino, Phys. Rev. Lett. 118 (2017) 031804 [arXiv: 1605.04299] [INSPIRE].

[43] P. Coloma, D.V. Forero and S.J. Parke, DUNE sensitivities to the mixing between sterile and tau neutrinos, JHEP 07 (2018) 079 [arXiv:1707.05348] [INSPIRE].

[44] S. Choubey, D. Dutta and D. Pramanik, Imprints of a light sterile neutrino at DUNE, T2HK and T2HKK, Phys. Rev. D 96 (2017) 056026 [arXiv: 1704.07269] [INSPIRE].

[45] S. Choubey, D. Dutta and D. Pramanik, Measuring the sterile neutrino CP phase at DUNE and T2HK, Eur. Phys. J. C 78 (2018) 339 [arXiv:1711.07464] [INSPIRE].

[46] S.K. Agarwalla, S.S. Chatterjee and A. Palazzo, Signatures of a light sterile neutrino in T2HK, JHEP 04 (2018) 091 [arXiv: 1801.04855] [INSPIRE].

[47] N. Haba, Y. Mimura and T. Yamada, On $\theta_{23}$ octant measurement in $3+1$ neutrino oscillations in T2HKK, arXiv:1812.10940 [INSPIRE].

[48] A. Donini and D. Meloni, The $2+2$ and $3+1$ four family neutrino mixing at the neutrino factory, Eur. Phys. J. C 22 (2001) 179 [hep-ph/0105089] [INSPIRE].

[49] A. Donini, M. Lusignoli and D. Meloni, Telling three neutrinos from four neutrinos at the neutrino factory, Nucl. Phys. B 624 (2002) 405 [hep-ph/0107231] [INSPIRE].

[50] A. Donini, M. Maltoni, D. Meloni, P. Migliozzi and F. Terranova, $3+1$ sterile neutrinos at the CNGS, JHEP 12 (2007) 013 [arXiv:0704.0388] [INSPIRE].

[51] A. Dighe and S. Ray, Signatures of heavy sterile neutrinos at long baseline experiments, Phys. Rev. D 76 (2007) 113001 [arXiv:0709.0383] [INSPIRE].

[52] A. Donini, K.-I. Fuki, J. Lopez-Pavon, D. Meloni and O. Yasuda, The discovery channel at the neutrino factory: $\nu_{\mu} \rightarrow \nu_{\tau}$ pointing to sterile neutrinos, JHEP 08 (2009) 041 [arXiv:0812.3703] [INSPIRE]. 
[53] O. Yasuda, Sensitivity to sterile neutrino mixings and the discovery channel at a neutrino factory, in Physics beyond the standard models of particles, cosmology and astrophysics. Proceedings, $5^{\text {th }}$ International Conference, Beyond 2010, Cape Town, South Africa, 1-6 February 2010, World Scientific, Singapore (2011), pg. 300 [arXiv: 1004.2388] [INSPIRE].

[54] D. Meloni, J. Tang and W. Winter, Sterile neutrinos beyond LSND at the neutrino factory, Phys. Rev. D 82 (2010) 093008 [arXiv: 1007.2419] [InSPIRE].

[55] B. Bhattacharya, A.M. Thalapillil and C.E.M. Wagner, Implications of sterile neutrinos for medium/long-baseline neutrino experiments and the determination of $\theta_{13}$, Phys. Rev. D 85 (2012) 073004 [arXiv:1111.4225] [INSPIRE].

[56] A. Donini, P. Hernández, J. Lopez-Pavon, M. Maltoni and T. Schwetz, The minimal $3+2$ neutrino model versus oscillation anomalies, JHEP 07 (2012) 161 [arXiv:1205.5230] [INSPIRE].

[57] R. Gandhi, B. Kayser, S. Prakash and S. Roy, What measurements of neutrino neutral current events can reveal, JHEP 11 (2017) 202 [arXiv:1708.01816] [INSPIRE].

[58] P.F. de Salas, D.V. Forero, C.A. Ternes, M. Tortola and J.W.F. Valle, Status of neutrino oscillations 2018: $3 \sigma$ hint for normal mass ordering and improved CP sensitivity, Phys. Lett. B 782 (2018) 633 [arXiv:1708.01186] [InSPIRE].

[59] F. Capozzi, E. Lisi, A. Marrone and A. Palazzo, Current unknowns in the three neutrino framework, Prog. Part. Nucl. Phys. 102 (2018) 48 [arXiv:1804.09678] [inSPIRE].

[60] I. Esteban, M.C. Gonzalez-Garcia, A. Hernandez-Cabezudo, M. Maltoni and T. Schwetz, Global analysis of three-flavour neutrino oscillations: synergies and tensions in the determination of $\theta_{23}, \delta_{C P}$ and the mass ordering, JHEP 01 (2019) 106 [arXiv:1811.05487] [INSPIRE].

[61] F. Capozzi, C. Giunti, M. Laveder and A. Palazzo, Joint short- and long-baseline constraints on light sterile neutrinos, Phys. Rev. D 95 (2017) 033006 [arXiv:1612.07764] [INSPIRE].

[62] S. Gariazzo, C. Giunti, M. Laveder and Y.F. Li, Updated global $3+1$ analysis of short-baseline neutrino oscillations, JHEP 06 (2017) 135 [arXiv:1703.00860] [INSPIRE].

[63] M. Dentler et al., Updated global analysis of neutrino oscillations in the presence of eV-scale sterile neutrinos, JHEP 08 (2018) 010 [arXiv:1803.10661] [INSPIRE].

[64] A. Diaz, C.A. Argüelles, G.H. Collin, J.M. Conrad and M.H. Shaevitz, Where are we with light sterile neutrinos?, arXiv:1906.00045 [INSPIRE].

[65] ESS $\nu \mathrm{SB}$ collaboration, A very intense neutrino super beam experiment for leptonic CP-violation discovery based on the European spallation source linac, Nucl. Phys. B 885 (2014) 127 [arXiv: 1309.7022] [INSPIRE].

[66] ESS $\nu$ SB PROJECT collaboration, The ESS $\nu S B$ project for leptonic CP-violation discovery based on the European Spallation Source Linac, Nucl. Part. Phys. Proc. 273-275 (2016) 1726 [INSPIRE].

[67] M. Dracos, The European Spallation Source neutrino super beam, in Proceedings, Prospects in Neutrino Physics (NuPhys2017), London, U.K., 20-22 December 2017, pg. 33 [arXiv: 1803.10948] [INSPIRE].

[68] ESS $\nu$ SB collaboration, Neutrino CP violation with the ESS neutrino super beam (ESSLSB), PoS (ICHEP2018) 524 (2019) [INSPIRE]. 
[69] E. Fernandez-Martinez, private communication, (2013).

[70] MEMPHYS collaboration, Study of the performance of a large scale water-Cherenkov detector (MEMPHYS), JCAP 01 (2013) 024 [arXiv:1206.6665] [INSPIRE].

[71] L. Agostino, private communication, (2013).

[72] S.K. Agarwalla, S. Choubey and S. Prakash, Probing neutrino oscillation parameters using high power superbeam from ESS, JHEP 12 (2014) 020 [arXiv:1406.2219] [INSPIRE].

[73] P. Huber, M. Lindner and W. Winter, Simulation of long-baseline neutrino oscillation experiments with GLoBES (General Long Baseline Experiment Simulator), Comput. Phys. Commun. 167 (2005) 195 [hep-ph/0407333] [INSPIRE].

[74] P. Huber, J. Kopp, M. Lindner, M. Rolinec and W. Winter, New features in the simulation of neutrino oscillation experiments with GLoBES 3.0: general Long Baseline Experiment Simulator, Comput. Phys. Commun. 177 (2007) 432 [hep-ph/0701187] [INSPIRE].

[75] J. Kopp, Sterile neutrinos and non-standard neutrino interactions in GLoBES, https://www.mpi-hd.mpg.de/personalhomes/globes/tools/snu-1.0.pdf, (2010).

[76] S.K. Agarwalla, S.S. Chatterjee, A. Dasgupta and A. Palazzo, Discovery potential of T2K and NOvA in the presence of a light sterile neutrino, JHEP 02 (2016) 111 [arXiv: 1601.05995] [INSPIRE].

[77] A.M. Dziewonski and D.L. Anderson, Preliminary reference earth model, Phys. Earth Planet. Inter. 25 (1981) 297.

[78] P. Huber, M. Lindner and W. Winter, Superbeams versus neutrino factories, Nucl. Phys. B 645 (2002) 3 [hep-ph/0204352] [INSPIRE].

[79] G.L. Fogli, E. Lisi, A. Marrone, D. Montanino and A. Palazzo, Getting the most from the statistical analysis of solar neutrino oscillations, Phys. Rev. D 66 (2002) 053010 [hep-ph/0206162] [INSPIRE].

[80] DUNE collaboration, Experiment simulation configurations used in DUNE CDR, arXiv: 1606.09550 [INSPIRE].

[81] Hyper-Kamiokande Working Group collaboration, A long baseline neutrino oscillation experiment using J-PARC neutrino beam and Hyper-Kamiokande, arXiv:1412.4673 [INSPIRE]. 